\title{
ON THE CONVERGENCE OF THE SERIES USED IN THE SUBJECT OF PERTURBATIONS.
}

BY. DR. G. W. HILL.

THE perturbations of the planets and the coördinates of the moon have been developed by astronomers in infinite series of terms involving sines or cosines of linear functions of two or more arguments with positive or negative integral multipliers. These arguments vary proportionally with the time, and their periods, in accordance with notions derived from the theory of probabilities, are supposed to be incommensurable with each other. Recently M. Poincaré has much insisted that, under the latter condition, these series, in the rigorous mathematical sense, are divergent (Les Méthodes Nouvelles de la Mécanique Céleste, Vol. II., pp. 277-280). However, the reasons brought forward to sustain this opinion are scarcely convincing, and I think there has been some scepticism among astronomers in reference to the matter. Without attempting to find any flaw in M. Poincaré's logic, I simply wish to point out a class of cases where the convergency of the series can be shown in spite of the incommensurability of the component arguments.

In many problems of dynamics, where the integral of conservation of areas has place, we shall often have the longitude $\lambda$ of the moving point given by a quadrature. We choose as our example the equation

$$
\frac{1}{\mathrm{n}} \frac{d \lambda}{d t}=\sum_{i=0}^{i=\infty} \sum_{i^{\prime}=-\infty}^{i^{\prime}=+\infty} \alpha^{i+\left|i^{\prime}\right|} \cos \left(i l+i^{\prime} l^{\prime}\right)
$$

in which $l=n t+c$ and $l^{\prime}=n^{\prime} t+c^{\prime}$, and $\alpha$ is a positive constant less than unity. Here $\lambda$ corresponds to M. Poincaré's $\log x$ (p. 279 of the above-quoted volume). Under the condition named, the series of (1) is convergent. Now let both members of the equation be integrated; putting $\mu$ for $\frac{n^{\prime}}{n}$, we
have

$$
\lambda=\epsilon+\mathrm{n} t+\frac{\mathrm{n}}{n} \Sigma \frac{1}{i+i^{\prime} \mu} \alpha^{i+\left|i^{\prime}\right|} \sin \left(i l+i^{\prime} \eta^{\prime}\right),
$$

$\epsilon$ being the added arbitrary constant, and the sign of summation $\Sigma$ having the same extension as that of the double sign in (1), except that the combination $i=i^{\prime}=0$ is omitted. When 
$\mu$ is an irrational quantity, the summation of this equation constitutes a divergent series according to M. Poincaré.

We prefer to write (2) in the more expanded form

$$
\begin{aligned}
\lambda=\epsilon+\mathrm{n} t & +\frac{\mathrm{n}}{n}\left\{\Sigma \frac{1}{i} \alpha^{i}\left[\sin i l+\frac{1}{\mu} \sin i l^{\prime}\right]\right. \\
& +2 \Sigma \Sigma \frac{i}{i^{2}-i^{12} \mu^{2}} \alpha^{i+i^{\prime}} \sin i l \cos i^{\prime} l^{\prime} \\
& \left.-2 \mu \Sigma \Sigma \frac{i^{\prime}}{i^{2}-i^{2} \mu^{2}} \alpha^{i+i^{\prime}} \cos i l \sin i^{\prime} l^{\prime}\right\},
\end{aligned}
$$

where the extent of the summation, in all cases, is from 1 to $+\infty$. This will be the signification of the sign $\Sigma$ hereafter.

Before we proceed to consider the question of convergence in reference to (3), it may be of interest to point out that the series of (1) admits of summation. For, by arranging it according to cosines of multiples of $l^{\prime}$, we have

$$
\begin{aligned}
& \frac{1}{\mathrm{n}} \frac{d \lambda}{d t}=\left[1+\alpha \cos l+\alpha^{2} \cos 2 l+\alpha^{3} \cos 3 l+\cdots\right] \\
& +\left[1+2 \alpha \cos l+2 \alpha^{2} \cos 2 l+2 \alpha^{3} \cos 3 l+\cdots\right] \alpha \cos l^{\prime} \\
& +\left[1+2 \alpha \cos l+2 \alpha^{2} \cos 2 l+2 \alpha^{3} \cos 3 l+\cdots\right] \alpha^{2} \cos 2 l^{\prime} \\
& + \text {. . . . . . . . . . . . . . . } \\
& =\left[1+\alpha \cos l+\alpha^{2} \cos 2 l+\cdots\right] \\
& +\left[1+2 \alpha \cos l+2 \alpha^{2} \cos 2 l+\cdots\right]\left[\alpha \cos l^{\prime}+\alpha^{2} \cos 2 l^{\prime}+\cdots\right] \\
& =\frac{1+\alpha^{4}-\alpha\left(1+\alpha^{2}\right)\left(\cos l+\cos l^{\prime}\right)+2 \alpha^{2} \cos l \cos l^{\prime}}{\left(1-2 \alpha \cos l+\alpha^{2}\right)\left(1-2 \alpha \cos l^{\prime}+\alpha^{2}\right)} \text {. }
\end{aligned}
$$

Then $\lambda$ can be expressed by the following quadrature:

$\lambda=\epsilon+\mathrm{n} t+$

$\overline{2} \int_{0}^{t}\left[\frac{\left(1-\alpha^{2}\right)^{2}}{\left[1-2 \alpha \cos (n t+c)+\alpha^{2}\right]\left[1-2 \alpha \cos \left(n^{\prime} t+c^{\prime}\right)+\alpha^{2}\right]}-1\right] d t$.

Our supply of functions in the integral calculus is inadequate to the expression of this quadrature in finite terms; but there is no bar to our finding the amount of motion of $\lambda$ between any two given times $t_{0}$ and $t_{1}$ by the process of mechanical quadratures.

Quadratures may also be invoked to aid in the expression of (3). For, by putting

$$
\begin{aligned}
& X_{i}=\frac{1}{1-i^{2} \mu^{2}} \alpha \cos l+\frac{1}{4-i^{2} \mu^{2}} \alpha^{2} \cos 2 l+\frac{1}{9-i^{2} \mu^{2}} \alpha^{3} \cos 3 l+\cdots, \\
& \text { as well as } \quad P_{i}=-\frac{d X_{i}}{d l}, \quad Q_{i}=i X_{i}
\end{aligned}
$$


(3) takes the form

$$
\begin{aligned}
\lambda=\epsilon & +\mathrm{n} t+\frac{\mathrm{n}}{n}\left\{\mathbf{\Sigma} \frac{1}{i} \alpha^{2}\left[\sin i l+\frac{1}{\mu} \sin i l^{\prime}\right]\right. \\
& +2\left(P_{1} \alpha \cos l^{\prime}+P_{2} \alpha^{2} \cos 2 l^{\prime}+P_{3} \alpha^{3} \cos 3 l^{\prime}+\cdots\right) \\
& \left.-2 \mu\left(Q_{1} \alpha \sin l^{\prime}+Q_{2} \alpha^{2} \sin 2 l^{\prime}+Q_{3} \alpha^{3} \sin 3 l^{\prime}+\cdots\right)\right\} .
\end{aligned}
$$

But it will be perceived that, putting

$$
L=\frac{\alpha \cos l-\alpha^{2}}{1-2 \alpha \cos l+\alpha^{2}}
$$

$X_{i}$ satisfies the linear differential equation of the second order

$$
\frac{d^{2} X_{i}}{d l^{2}}+i^{2} \mu^{2} X_{i}+L=0 .
$$

By the integration of this, we have

$$
X_{i}=\cos (i \mu l) \int L \sin (i \mu l) d l-\sin (i \mu l) \int L \cos (i \mu l) d l .
$$

The first summation of (6) may be obtained through the use of the well-known equation

$$
\Sigma \frac{1}{i} \alpha^{i} \sin i l=-\frac{1}{2} l+\arctan \left[\frac{1+\alpha}{1-\alpha} \tan \frac{l}{2}\right] \text {. }
$$

We come now to the consideration of the question of convergence of the two double summations in (3). In these we may put

$$
\sin i l=\cos i l=\sin i^{\prime} l^{\prime}=\cos i^{\prime} l^{\prime}=1 ;
$$

the matter at issue is not thereby changed. Hence it suffices to determine the convergence or divergence of the two series

$$
\Sigma \Sigma \frac{i}{i^{2}-i^{\prime 2} \mu^{2}} \alpha^{i+i^{\prime}}, \quad \Sigma \Sigma \frac{i^{\prime}}{i^{2}-i^{\prime 2} \mu^{2}} \alpha^{i+i^{\prime}}
$$

It is necessary now to specify the precise nature of the quantity $\mu$. As an example, we assume that $\mu=\sqrt{h}, h$ being a non-square integer. The divisor in the two series is then $i^{2}-h i^{2}$. From the theory of indeterminate equations of the second degree, we learn that the least absolute value of this expression is unity; that is, we may write

$$
\left|i^{2}-h i^{\prime 2}\right| \geqq 1 \text {. }
$$

Unity may therefore be substituted for this divisor in the summations just given without thereby modifying the question of their convergence, which is thus narrowed to the convergence or divergence of the single expression

$$
\Sigma \Sigma i \alpha^{i+i^{\prime}} \text {. }
$$


But this series is convergent, being equivalent to the product

$$
\left[\Sigma i \alpha^{i}\right]\left[\Sigma \alpha^{i}\right]=\left[\alpha+2 \alpha^{2}+3 \alpha^{3}+\cdots\right]\left[\alpha+\alpha^{2}+\alpha^{3}+\cdots\right] .
$$

If we agree to take $j$ terms of each factor of this as an approximation, the error committed will be

$$
\frac{j(1-\alpha)+2}{(1-\alpha)^{3}} \alpha^{j+2},
$$

which may be made as small as we please by taking $j$ sufficiently large. This expression is also a superior limit to the error committed in either of the double summations of (3) when the series is pushed to the same extent in reference to the varying integers $i$ and $i^{\prime}$. take

As a more general example, including the former, we will

$$
\mu^{2}=\frac{p}{q}+\sqrt{\frac{p^{\prime}}{q^{\prime}}}
$$

where $p, q, p^{\prime}$, and $q^{\prime}$ are integers, and $\frac{p^{\prime}}{q^{\prime}}$ is not an exact square. If we substitute this value of $\mu^{2}$ in the expression $i^{2}-i^{\prime 2} \mu^{2}$, and rationalize and render integral this denominator, multiplying it by the proper factor, we find that it becomes

$$
q^{\prime}\left(q^{2} i^{2}-p^{2} i^{\prime 2}\right)^{2}-p^{\prime} q^{4} i^{\prime 4}
$$

Now this expression, which is integral, cannot vanish, for this would make $\sqrt{\frac{p^{\prime}}{q^{\prime}}}$ rational; consequently, its absolute value is at least unity. We may then substitute unity for it in the summations we consider without affecting the question of their convergence. Thus, the latter is seen to depend on the convergence of

$$
\Sigma \Sigma i^{3} \alpha^{i+i^{\prime}} \text { and } \Sigma \Sigma i^{2} i^{\prime} \alpha^{i+i^{\prime}} \text {. }
$$

As these summations are quite plainly convergent, there is nothing further to be said.

As a still greater generalization, let us suppose that $\mu$ is an irrational root of an algebraic equation with rational coefficients. Then, in a similar way as before, $i^{2}-i^{\prime 2} \mu^{2}$ may be rationalized and rendered integral by multiplying by the proper factor. The absolute value of the thus modified denominator is at least unity. On consulting the form of the numerator, it is gathered that the convergence of our series depends on that of various summations whose general type is

$$
\Sigma \Sigma i^{\nu} i^{\prime v^{\prime}} \alpha^{i+i^{\prime}}
$$


where $v$ and $v^{\prime}$ are finite positive integers. The convergence is therefore established.

When $\mu$ is rational, we would simply transfer the terms which, in the integration, become proportional to $t$ to the term $\mathrm{n} t$ of (3). Then the remainder would constitute a periodic and convergent series. Thus, in all cases where $\mu$ is a root of an algebraic equation with rational coefficients, (3) may be affirmed to be a convergent series.

Our method of treatment cannot be applied in the case where $\mu$ is a root of a transcendental equation. But it may be remarked that the higher the degree of the equation which has $\mu$ for a root, the larger become the exponents $\nu$ and $\nu^{\prime}$. Thus, one is led to think that, when $\mu$ is a root of a transcendental equation, these exponents become infinite. Should this be correct, the summations, whose general type has just been given, become divergent. But we would not be warranted in concluding thence the divergence of (3). The whole question turns on the properties of the integral

$$
\int\left[\frac{\left(1-\alpha^{2}\right)^{2}}{\left[1-2 \alpha \cos (n t+c)+\alpha^{2}\right]\left[1-2 \alpha \cos \left(n^{\prime} t+c^{\prime}\right)+\alpha^{2}\right]}-1\right] d t .
$$

It is possible that the ratio $\frac{n^{\prime}}{n}$ may have values which would make this expression tend towards infinity as the limits of integration were removed farther from each other. But I am not aware that this has been proved. But it is something gained to have established that, when $\frac{n^{\prime}}{n}$ is an irrational root of an algebraic equation with rational coefficients, the expression is always contained between finite limits, whatever may be the limits of integration.

Our conclusions still hold when in (1) we substitute the general coefficient $C_{i, i^{\prime}}$ for $\alpha^{i+\left|i^{\prime}\right|}$, provided we have the condition

$$
\left|C_{i, i^{\prime}}\right| \bar{\Sigma} \alpha^{i+\left|i^{\prime}\right|}
$$

$\alpha$ being positive and less than unity. Also, we might assume a different rate of decrement in the coefficients with augmenting multiples of $l^{\prime}$ from that which belongs to $l$. Calling this $\alpha^{\prime}$, for $\alpha^{i+\left|i^{i}\right|}$ we should have $\alpha^{i} \alpha^{\left|i^{i}\right|}$, and the course of reasoning would be scarcely changed by this modification. In case there are more than two elementary arguments, the mode of proceeding is quite similar. The ratios $\frac{n^{\prime}}{n}, \frac{n^{\prime \prime}}{n}, \frac{n^{\prime \prime \prime}}{n}$, etc., being irrational roots of algebraical equations, the divisors introduced by integration must be rationalized and rendered integral by multiplying both terms of the fraction by the proper factor. The convergence of the series is made out as before. 